\title{
Dual wavelength optical system for multiple quantum communication transmitters in Cubesat platform
}

Christian Menninger, Florian Moll, Benjamin Rödiger

Christian Menninger, Florian Moll, Benjamin Rödiger, "Dual wavelength optical system for multiple quantum communication transmitters in Cubesat platform," Proc. SPIE 11852, International Conference on Space Optics ICSO 2020, 118525M (11 June 2021); doi: 10.1117/12.2599965

SPIE Event: International Conference on Space Optics - ICSO 2021, 2021, Online Only 


\section{International Conference on Space Optics-ICSO 2020}

Virtual Conference

30 March-2 April 2021

Edited by Bruno Cugny, Zoran Sodnik, and Nikos Karafolas
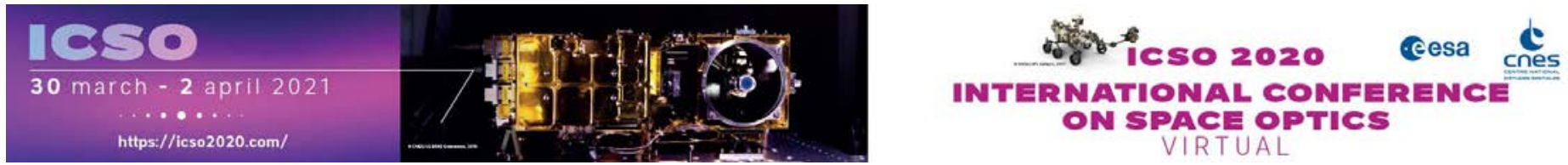

\section{Dual Wavelength Optical System for Multiple Quantum Communication Transmitters in Cubesat Platform}

\section{Cesa tisprestatast ecnes}




\title{
Dual Wavelength Optical System for Multiple Quantum Communication Transmitters in Cubesat Platform
}

\author{
Christian Roubal ${ }^{\mathrm{a}}$, Florian Moll ${ }^{\mathrm{a}}$, and Benjamin Rödiger ${ }^{\mathrm{a}}$ \\ ${ }^{a}$ German Aerospace Center - Institute of Communications and Navigation, Münchener Str. 20, \\ 82234 Weßling, Germany
}

\begin{abstract}
Quantum Key Distribution systems will play an important role in future networks for secure data communication. In order to provide a global coverage of a future Quantum Key Distribution service, satellites will be needed to bridge large distances. These satellite systems must be cost efficient to facilitate deployment since many network nodes will be needed. The CubeSat standard is frequently used for New Space projects as a versatile platform for satellite payloads. It is also chosen as a baseline for the construction of the system described in this paper. The DLR Institute of Communications and Navigation develops optical free space communication systems for scientific research in classical and quantum communications. In the OSIRIS4CubeSat (Optical Opace Infrared Downlink System for CubeSats) project a $1 / 3$ U CubeSat laser-communication terminal for up to $100 \mathrm{Mbit} / \mathrm{s}$ downlinks was developed. ${ }^{1}$ This developement is adapted to be used for quantum communications tests with multiple transmitters in the scope of the project $Q U B E .^{2}$ Since the OSIRIS4CubeSat terminal was designed for C- and L-band wavelengths, a redesign of the optical system is needed to achieve polychromatic performance for C- and L-band and $850 \mathrm{~nm}$. The optical system consists of a fiber collimator, a fine pointing assembly and an afocal telescope. Most important requirement of the latter is a similar magnification for all wavelengths to ensure coaligned beams pointing to the optical ground station (OGS). As the afocal telescope is used bidirectionally, it also needs to be optimized for the incident wave-front of the beacon laser from the OGS with respect to the beam shape at the tracking sensor which is used in the fine pointing assembly. These parameters are important for a correct pointing control. In addition to the laser terminal, a fiber-based wavelength division multiplexer (WDM) was specified for combining signals from three sources into one output fiber. It is based on cascaded thin film interference filters which are coupled to the fibers. Especially the propagation of $850 \mathrm{~nm}$ and C/L-band signals in one single mode fiber is critical. Therefore the types of optical fibers were selected with respect to the bend loss, to single mode propagation behaviour, polarisation integrity and optimal cladding diameter for production.
\end{abstract}

Keywords: CubeSat, Quantum Key Distribution, Free-Space Optical Communications, New Space, Laser Terminal

\section{INTRODUCTION}

Optical communications is already well established for high speed and long distance data transfer. It also expands evermore towards space, either for high speed optical downlinks to transmit data created on a satellite or as a network node to relay data. ${ }^{3,4}$ Optical links can also provide an infrastructure for future secure communications using Quantum Key Distribution (QKD). Experiments on ground with fibre to fibre connections were already frequently utilised for demonstrating this technique and also showing the limitation in transmission range due to the attenuation in fibres. Thus, free space links can help to overcome this limitation by using satellites for large distances. QKD experiments on satellite platforms or also aircraft platforms are rare. ${ }^{5,6}$ The reason for this might be the costs of a satellite launch as well as the risk of failure due to the flight readiness of the components used for a QKD payload and the costs for developement and qualification thereof. However, as the New Space approach leads to more cost effective satellites using Commercial Off-The-Shelf (COTS) components

Further author information: (Send correspondence to C.R.)

C.R.: E-mail: chrisitan.roubal@dlr.de, Telephone: +49 8153281114

B.R.: E-mail: benjamin.roediger@dlr.de, Telephone: +498153282944 
as well as smaller payloads, the developement and tests of QKD payloads are getting more attractive, especially for research facilities. DLR's OSIRIS4CubeSat terminal payload provides a basis for the adaption to serve as a terminal for multiple QKD sources beside the already integrated classical communications system. As the optical system of the predecessor is designed for C- and L-band wavelengths, it needs to be redesinged for the additional use of $850 \mathrm{~nm}$ which is one of the quantum sources. Reusability of already qualified components as well as the application of COTS components were of particular interest to create a low-cost and rapidly developable terminal. Hence, several considerations must be taken into account in order to use multiple wavelengths in the optical system. The evolution of the main optical terminal design and the principle of wavelength combination from the three payloads, two quantum and one classical, are decribed in Section 2 and 3, respectively.

\section{OPTICAL DESIGN OF THE LASER TERMINAL}

\subsection{Design Considerations}

The starting point of the optical and mechanical design is the OSIRIS4CubeSat payload, which is shown as a CAD model in Figure 1. It consists of two main functional systems: The transmitter system (Tx-system) and receiver system (Rx-system). In the Tx-system, light is coupled from a fibre into a free space collimated beam, which is magnified with an afocal telescope after being reflected by a beam splitter and a Fast Steering Mirror (FSM). The Rx-system receives a laser beacon signal sent from an OGS and compresses the incident beam with the same telescope used in the Tx-system. It also passes the FSM, transmits through the beam splitter and is directed to a four quadrant detector (4QD). This system enables the detection of the angular offset between the boresight of the satellite optical sytem and that of the OGS.

The assigned numbers in Figure 1 are indicating main components of the system and shall be briefly summarised in the following in order to understand the boundary conditions of the optical design for further developement: Two assembled solid aluminium blocks provide a rigid and stiff basis (1) for the whole system. This includes the aperture lens (2) and the rest of the optical assembly which is coloured in red. The mainboard PCB (3) is directly attached to the blocks. The laser (4) is connected via FC/APC plug to the collimator (5), which enables angular adjustment of the Tx-system to the Rx-system (Tx/Rx-alignment). The laser driver is located next to the laser and must be passively cooled with a heat sink (6). Since the accuracy of the satellite body pointing is limited and cannot always fulfill the requirements of the needed precision for an optical terminal, an optical tracking system is implemented. This consists of the FSM and the 4QD (7) working in a closed control loop. In order to minimise the effort for a redesign and qualification, several design considerations must be taken into account:

1. Fit everything inside the existing mechanical system without major changes.

2. Optimal pointing accuracy/maintaining pointing accuracy

3. Minimise aberrations due to transmission at an angular offset from the optical axis.

4. Lowest possible divergence for all Tx-wavelengths.

5. Matched magnification of the telescope and the payload's Angle Of Exitance (AOE) for all Tx-wavelengths.

Even though some of the above listed design criteria were already accounted for in the optical design of the OSIRIS4CubeSat payload, the performance has to be maintained and some new considerations appeared due to the additional wavelength and are interdependently. Criterion \#1 is the most design-influencing one, as it defines the maximum allowed size of the new optical system, as well as the beam paths for the Tx- and Rx-system. Criterion \#2 remains the same, but is stronly related to criterion \#3, since the Tx- and Rx-systems partly use the same optical path. Therefore the Rx-system also depends passively on the new wavelength. There, precise and continous tracking is only possible, if the spot shape at the 4QD is radial symmetric, the optical power homogenously distributed and the spot size appoximately between $300 \mu m$ and $600 \mu m$ in diameter. Furthermore, criteria \#4 and \#5 are important for multiple optical downlinks at the same time, i.e. tracking is performed with a L-band wavelength and the downlinks are performed with one or both of the C-band wavelengths as well as with $850 \mathrm{~nm}$. 


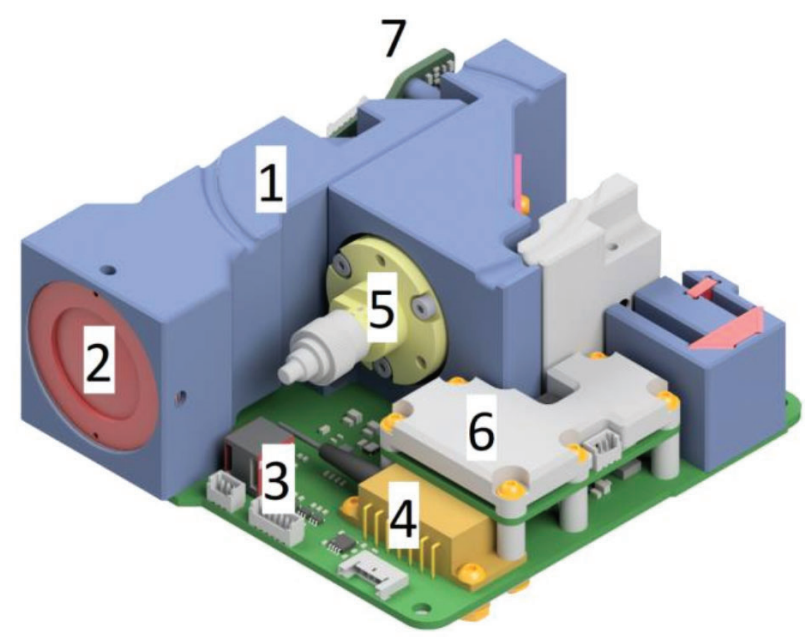

Figure 1. CAD model of the OSIRIS4CubeSat payload. The numbers are indicating the main components; 1: main body, 2: aperture lens, 3: mainboard, 4: laser, 5: fibre collimator, 6: heat sink for laser driver, 7: 4QD. ${ }^{1}$

For an unaberrated system using ideal optical elements, the optical paths from inside the satellite to earth would be the same for all wavelengths, even if all beams were transmitted at an angular offset to the telescope's optical axis which normally induces aberrations. This ideal scenario is shown in Figure 2a).

A more realistic scenario is shown in Figure $2 \mathrm{~b}$ ). The divergences are not perfectly matched due to residual lens errors and optomechanical offsets which might not be completely compensated or due to a thermal drift.

A worst case scenario is demonstrated in Figure 2c). This scenario must be avioded at all cost, since it is likey that a downlink for all wavelengths at the same time is not even possible. 2c) shows the situation in which the divergences are mismatched, the AOE for the two downlink beams is not matched and thus pointing performance is poor.

\section{a)}

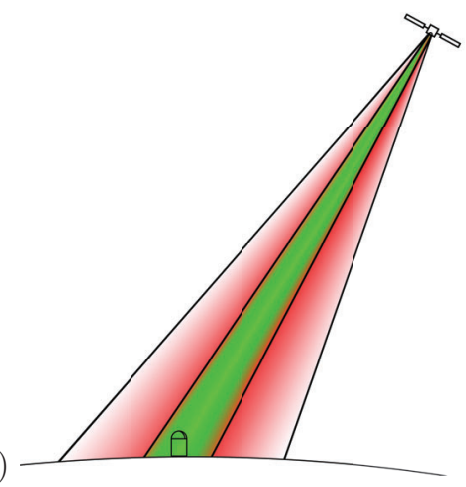

b)

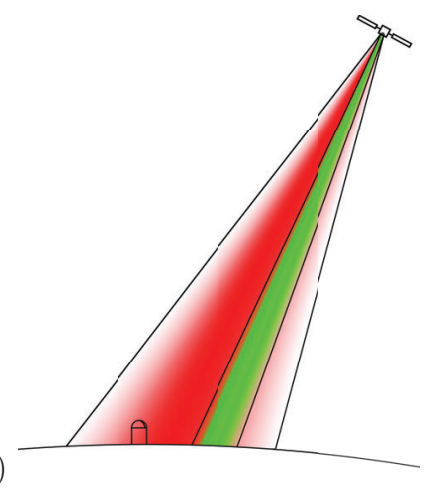

c)

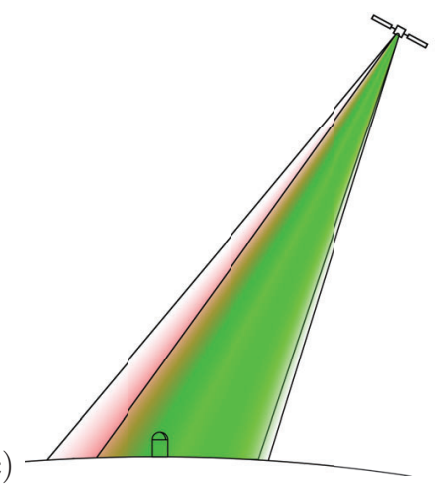

Figure 2. Different pointing scenarios from satellite to OGS using two different wavelengths: a) Optimal divergences and concentric beams, b) Optimal divergences, but angular offset and c) Angular offset with adapted divergences.

\subsection{Optical Design}

The optical design of the OSIRIS4CubeSat payload is taken as a basis and is created using optics design software Zemax. As only the Tx-wavelengths are changed, the Rx-system is left untouched. A first analysis of the optical properties shows that a redesign of all lenses for the Tx-system is necessary. Therefore, optimisation criteria 
are adopted to the new wavelengths and with that, the above mentioned criteria have to be adapted as well. The originally used three singlet lens system is split into a system of three achromatic doublets after verifying the feasibility of less achromatic doublets. However, the compensation of chromatic aberrations is not possible without simultaneously inducing spherical aberrations and coma in the telescope.

A cross sectional view of the final optical design model for the Tx-system is shown in Figure 3 . The rays indicate the beam paths at $0^{\circ} \mathrm{AOE}$ (blue) and $0.6^{\circ} \mathrm{AOE}$ (magenta) at C-band wavelength. The lens elements are indicated from L1 to L6 beginning with the lens L1, the first element of the achromatic doublet, which collimates the light exiting the optical fibre. Since the elements at the optical path from the fibre tip to L2 are static in their position and aligned to the optical axis, aberrations are lower compared to those occuring in the telescope. It was therefore sufficient to use spherical surfaces for L1 and L2. Since the beam splitter (BS) combines/divides the Tx- and Rx-system, each component in the shared beam path is optimised with respect to the optical properties of both systems. While the FSM is steering the Tx-beam, strong aberrations are induced if the beam path is not on the optical axis. The magenta-coloured rays in Figure 3 show the beam path for an $\mathrm{AOE}$ of $0.6^{\circ}$ which corresponds to an Angle Of Incidence (AOI) of $4.9^{\circ}$ to a plane perpendicular to the boresight of L3. As not only the AOI on L4 changes but also the position at which the beam is incident on its surface, the induced wavefront errors can be corrected if the shape of the surface was manipulated locally. The induced errors are radially symmetric and correctable with an aspheric shape of one of the surfaces of L3. Different AOI were taken into account for the optimisation process of the lens surfaces in order to assure a reasonable quality not only for the maximum achievable angle, but also for angles in between this range. Aspheric surfaces are more complex and expensive to manufacture and also take more time in the optimisation process. For that reason, all surfaces are spherical in the initial setup of the design process. The surface types for L3-L6 were changed one after another from spherical to aspherical, until a reasonable compromise between quality and cost was found. A market research for stock lenses also showed a potential replacement for L6 which was successfully implemented, as it was also available as an uncoated version. In the final design L6 is an ashperic stock lens; L1, L2 and L5 are custom made spherical lenses; and L4 and L3 are custom made aspherical lenses.

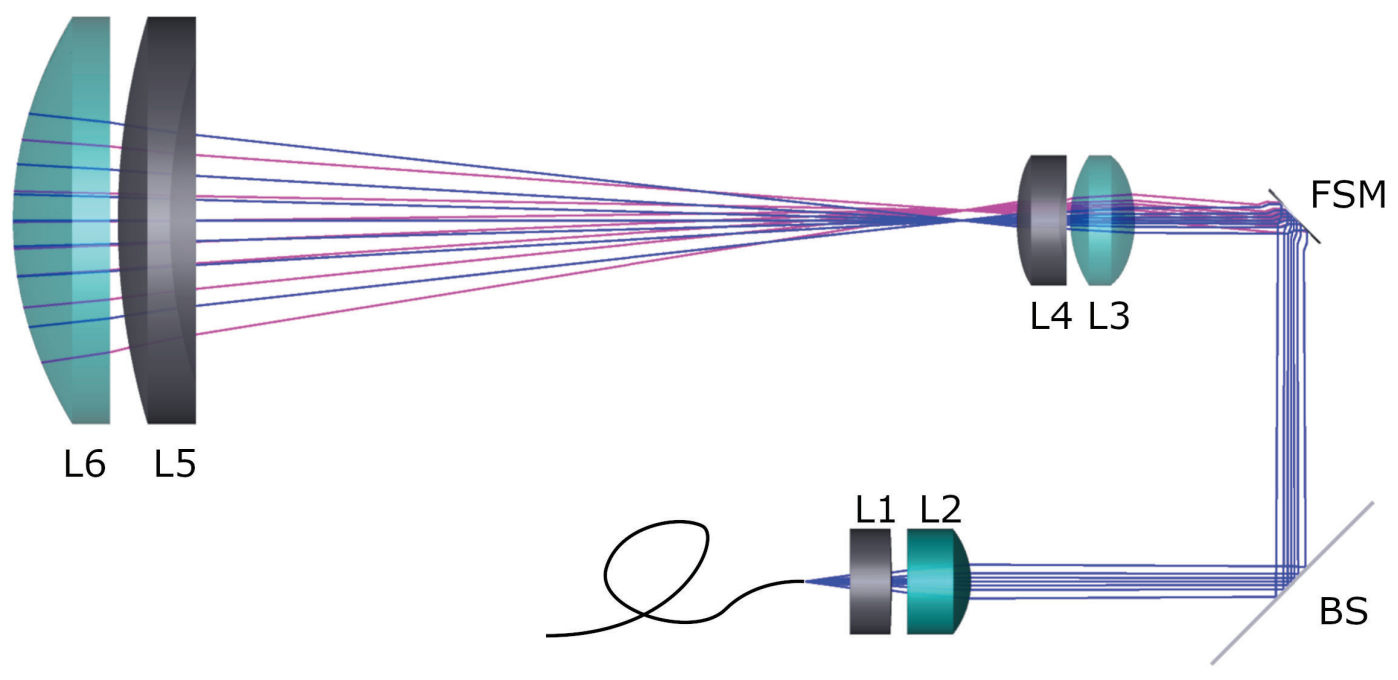

Figure 3. Cross sectional view of the optical Tx-system. The rays indicate the beam paths at $0^{\circ}$ AOE (blue) and $0.6^{\circ}$ AOE (magenta). The optical elements are indicated as follows: Beam Splitter (BS), Lens 1-6 (L1-6), Fine Steering Mirror (FSM).

The selection of the lens material is first done by comparing different flint and crown glasses according to their amount of radiation induced darkening from the ESA RADGLASS catalogue in which data is partly published. ${ }^{7}$ 
However, after first optimisation it turns out that the radius of curvature of some of the lenses becomes too small for machining a reasonable surface quality, if they were even manufacturable. In particular, the material of L1-L5 has to be changed. Only materials with a high refractive index come into account, so that the radius of curvature can be made larger, while the focal length of the lens is unchanged. For the correction of chromatic aberrations, an achromatic doublet is built by two lens elements per lens. L1, L4 and L5 have a negative focal lengths and are made of a high dispersive material. As the dispersion of standard glass materials is low in the near infrared spectrum, a chalcogenide glass is selected for these lens elements. For the positive focal lengths lens elements L2 and L3, a heavy flint glass is selected as the dispersion is low compared to the chalcogenise glass, but the refractive index is high compared to standard crown glass. In order to minimise back reflections, all lenses are equipped with a custom made anti reflection coating.

Contrary to the complete redesign of the Tx-system including the shared part of the telescope, the optical design of the Rx-system is completely reused. A cross sectional view of the complete optical system is shown in Figure 4. The blue and red rays indicate the beam paths of the Tx- and Rx-system, respectively. Beside the shared beam path, the Rx-system consits of a plano-convex spherical lens (L7) which projects the received beam onto the 4QD with the desired diameter of $\sim 600 \mu \mathrm{m}$. A prism is used to fold the beam for saving space and a filter (FLT) is placed in the beam path to filter out any stray light of different wavelength so that only the beacon wavelength is transmitted onto the 4QD. As parts of the optics and mechanics could be reused, engineering time and costs were minimised as well as risk of failure during the qualification phase.

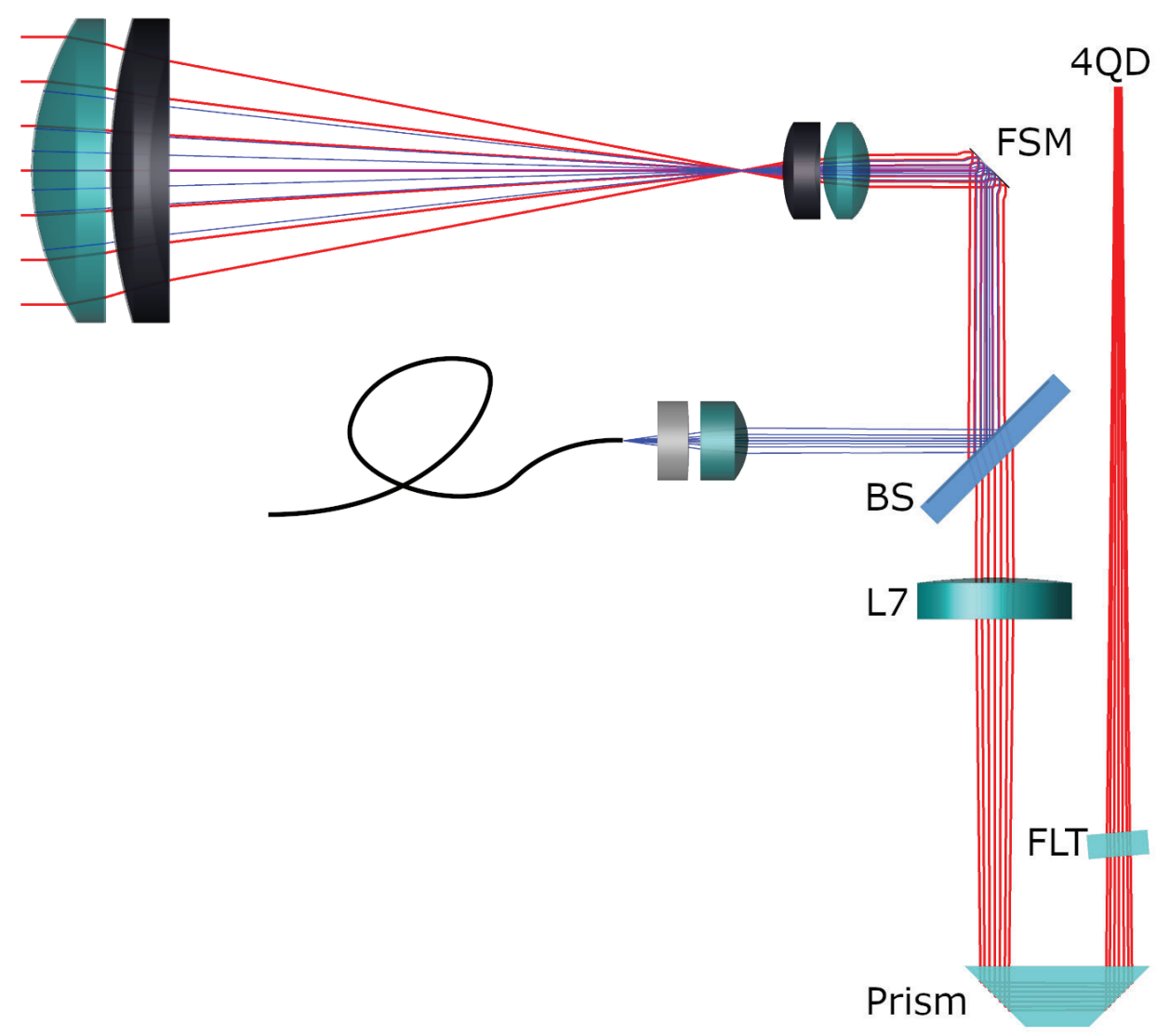

Figure 4. Cross sectional view of the optical Tx- and Rx-system. Rays to indicate the beam paths for the Tx- and Rx-System in blue and red, respectively. Optical elements: Lens 7 (L7), filter (FLT), Beam Splitter (BS), Fine Steering Mirror (FSM), Four Quadrant Diode (4QD). 


\section{WAVELENGTH DEVISION MULTIPLEXER}

The combination of multiple sources with different wavelengths into one optical path is often performed with free space dichromatic beam splitters. However, it would require a precise alignment between the optical systems of the payloads inside the satellite on one hand, and it would also require additional space in the optomechanical system from the reused OSIRIS4CubeSat payload. Thus, a wavelength combination based on free space coupling is out of option. A compact fibre based Wavelength Devision Multiplexer (WDM) is used instead. It is equipped with three FC/APC input fibre ports for the channels 1-3 and has a pigtailed FC/APC fibre output. Channel 1 and 2 are in the C- and L-band, therefore a fused fibre based filter cannot not be used. A thin film based micro-optical filter is built in instead which limits the size of the overall filter network to the minimal applicable bending radius of output fibre. Figure 5 shows an image of the WDM on the left hand side and a corresponding functional diagram on the right hand side. It consists of two cascaded WDMs, each using a microlens and a dichromatic beam splitter fixed in a glass tube to couple light from two input fibres into one output fibre. In the first filter stage all fibres are of the type Corning $Z B L$ which ensures an overall low bend loss and is compatible to the fibres used for channel 1 and 2. As the second stage combines the C-band and L-band wavelengths from the first stage with the $850 \mathrm{~nm}$ light of channel 3 in the second stage, three different fibres are used. While two fibres of the WDM's input were selected according to their matching propagation constant, the output fibre of the whole WDM needs to guide a broad range of wavelengths. As the type of all fibres need to be single mode, a compromise between propagation constant and bend loss is made. A market research results in a selection of two high index optical fibres, namely the Corning HI1060 and Corning HI980, which are both frequently used in EDFAs and DWDM networks. However, the cut-off wavelength of both fibres is above $850 \mathrm{~nm}$, smaller bending radii than given in the data sheet needs to be taken into account. Therefore, measurements of the output beam profile at $850 \mathrm{~nm}$ and bend loss in the C-band at different radii are performed for the selection between the two types of fibre. The best compromise is given by the HI980 fibre.
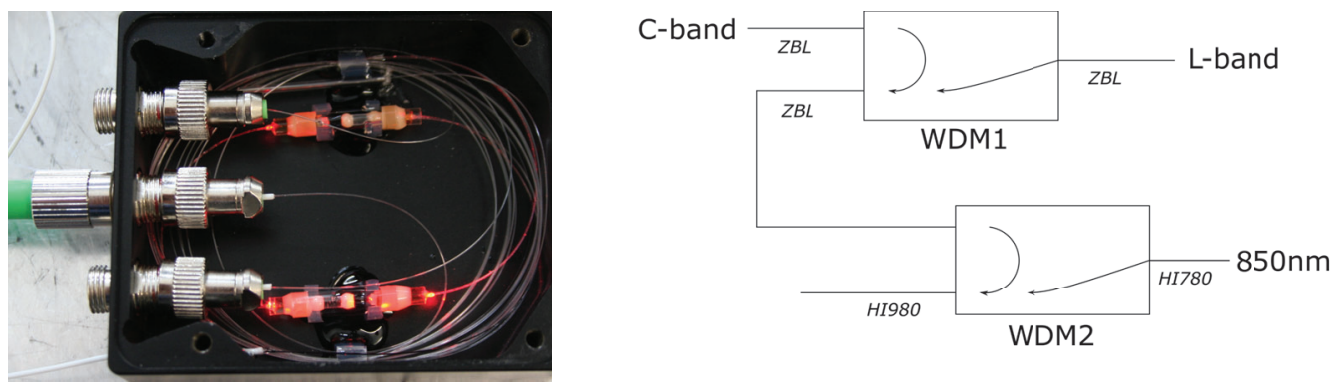

Figure 5. Left: Image of the inside of the WDM box. Right: Functional diagram of the two cascaded WDM filters.

\section{CONCLUSION}

An optical system for a laser terminal and a WDM has been developed as part of a satellite payload. The system is based on a previous design of a laser communication terminal and has to be optimised for the use of three sources emitting in the C- and L-band and at $850 \mathrm{~nm}$. A major design effort is the optimisation for matched divergences and a low angluar beam displacement for different AOEs within the space given by the mechanical dimensions of the OSIRIS4CubeSat payload. The combination of an infrared glass material and a heavy flint glass for spherical and aspherical lenses are the key parameters for a solution of the previously mentioned criteria.

\section{ACKNOWLEDGEMENTS}

This project is funded by the German Federal Ministry of Education and Research (BMBF) with the indication 16KIS0769. We thank the consortium partners LMU Munich, MPL Erlangen, OHB Systems AG, ZfT and Bitline System Pty Ltd. for fruitful discussions and support. 


\section{REFERENCES}

[1] Rödiger, B., Menninger, C., Fuchs, C., Grillmayer, L., Arnold, S., Rochow, C., Wertz, P., and Schmidt, C., "High data-rate optical communication payload for CubeSats," in [Laser Communication and Propagation through the Atmosphere and Oceans IX], Wayne, D. T., Anguita, J. A., and Bos, J. P., eds., 3, SPIE (24.08.2020 - 28.08.2020).

[2] Haber, R., Garbe, D., Busch, S., Rosenfeld, W., and Schilling, K., "Qube - a cubesat for quantum key distribution experiments." https://digitalcommons . usu. edu/cgi/viewcontent. cgi?article=4081\&context= smallsat (2018).

[3] Fujiwara, Y., Mokuno, M., Jono, T., Yamawaki, T., Arai, K., Toyoshima, M., Kunimori, H., Sodnik, Z., Bird, A., and Demelenne, B., "Optical inter-orbit communications engineering test satellite (OICETS)," Acta Astronautica 61(1-6), 163-175 (2007).

[4] Giggenbach, D., Fuchs, C., Schmidt, C., Jaiswal, R., Shrestha, A., and Keim, J., "Optical data downlinks from osiris on flying laptop satellite," (09 2019).

[5] Nauerth, S., Moll, F., Rau, M., Fuchs, C., Horwath, J., Frick, S., and Weinfurter, H., "Air-to-ground quantum communication," Nature Photonics 7(5), 382-386 (2013).

[6] Liao, S.-K., Cai, W.-Q., Liu, W.-Y., Zhang, L., Li, Y., Ren, J.-G., Yin, J., Shen, Q., Cao, Y., Li, Z.-P., Li, F.Z., Chen, X.-W., Sun, L.-H., Jia, J.-J., Wu, J.-C., Jiang, X.-J., Wang, J.-F., Huang, Y.-M., Wang, Q., Zhou, Y.-L., Deng, L., Xi, T., Ma, L., Hu, T., Zhang, Q., Chen, Y.-A., Liu, N.-L., Wang, X.-B., Zhu, Z.-C., Lu, C.-Y., Shu, R., Peng, C.-Z., Wang, J.-Y., and Pan, J.-W., "Satellite-to-ground quantum key distribution," Nature 549(7670), 43-47 (2017).

[7] Bézy, J.-L., Manolis, I., Vink, R., and Meynart, R., "The ESA radglass activity: a radiation study of non rad-hard glasses," in [International Conference on Space Optics - ICSO 2016], Karafolas, N., Cugny, B., and Sodnik, Z., eds., 62, SPIE (18.10.2016 - 21.10.2016). 